\title{
Biologia e tabela de vida de fertilidade de Hypercompe indecisa em dieta artificial
}

\author{
Dori Edson Nava(1), Gabriela Inés Diez-Rodríguez ${ }^{(1)}$, Mirtes Melo(1) e Ana Paula Schneid Afonso(1) \\ (1)Embrapa Clima Temperado, Caixa Postal 403, CEP 96001-970 Pelotas, RS. E-mail: nava@cpact.embrapa.br, gidiez@gmail.com, \\ mirtes@cpact.embrapa.br, anapaula@cpact.embrapa.br
}

\begin{abstract}
Resumo - O objetivo deste trabalho foi estudar a biologia de Hypercompe indecisa e construir a tabela de vida de fertilidade em dieta artificial, para criação em laboratório. Foram determinados: duração e sobrevivência das fases de ovo, larva e pupa; número de estádios larvais; razão sexual; peso de pupas; longevidade; fecundidade; e períodos de pré-oviposição e oviposição. Os períodos embrionário, larval e pupal foram de 6, 25,4 e 64,3 dias, com sobrevivência de 92,7, 92 e 71,9\%, respectivamente. Foram observados seis estádios larvais com durações variáveis. O peso de pupas foi: 1,04 g para fêmeas e 0,726 g para machos. O ciclo biológico foi de 95,6 dias com sobrevivência total de $61,3 \%$. As fêmeas colocaram, em média, 1.531 ovos durante 8,3 dias, e com um período de pré-oviposição de dois dias. A longevidade média de machos e fêmeas foi de 21,9 e 21,8 dias, respectivamente, e a razão sexual de machos para fêmeas foi 0,54 . A espécie $H$. indecisa aumentou 283 vezes a cada geração, e a duração média de uma geração é de 98 dias, e a razão finita de aumento é de 1,0593. A dieta artificial utilizada foi adequada para a criação de $H$. indecisa, em laboratório.
\end{abstract}

Termos para indexação: Arctiidae, bioecologia, praga de fruteiras, praga do milho, técnica de criação.

\section{Biology and fertility life table of Hypercompe indecisa on artificial diet}

\begin{abstract}
The objective of this work was to study the biology of Hypercompe indecisa and construct a life table of fertility on an artificial diet, aiming at insect rearing in the laboratory. The following variables were determined: duration and survival of egg, larval and pupal phases; number of larval stages; sexual ratio; pupal weight; longevity; fecundity; and the period of pre-oviposition and oviposition. The embrionary, larval and pupal phases were 6, 25.4 and 64.3 days, with survival of 92.7, 92 and 71.9\%, respectively. Six larval stages with variable durations were observed. Pupal weight was $1.04 \mathrm{~g}$ for females and $0.726 \mathrm{~g}$ for males. The biological cycle was 95.6 days, with $61.3 \%$ total survival. Females laid in average 1,531 eggs, during 8.3 days, with a pre-oviposition period of two days. The average longevity of males and females was 21.9 and 21.8 days, respectively, and the sexual ratio of males to females was 0.54 . Increase of $H$. indecisa was 283 times through each generation, with the generation average duration of 98 days, and the finite ratio of increase of 1.0593 . The used artificial diet was adequate for rearing H. indecisa, in the laboratory.
\end{abstract}

Index terms: Arctiidae, bioecology, fruit tree pest, corn pest, rearing technique.

\section{Introdução}

Na agricultura brasileira, os lepidópteros constituem a ordem de insetos com maior número de espécies nocivas, cuja fase larval pode danificar todas as partes das plantas cultivadas (Gallo et al., 2002). Pelo menos 21 espécies associadas ao milho (Zea mays L.) são conhecidas, distribuídas nas famílias Cosmopterigidae, Crambidae, Noctuidae, Pyralidae, Saturnidae e Arctiidae, e esta última é representada pela espécie Hypercompe indecisa (Walker, 1855) (Silva et al., 1968; Sanchez Soto \& Nakano, 2003).
A espécie $H$. indecisa tem sido relatada como praga das folhas e da espiga do milho, entretanto, nos últimos anos, também tem causado dano à fase inicial da cultura. As lagartas se alimentam na base das plântulas, próximo ao solo e causam seu tombamento. Além do milho, também se alimentam de folhas de mais de 20 hospedeiros, entre eles as hortaliças batatinha (Solanum tuberosum L.), couve (Brassica oleracea L.) e alface (Lactuca sativa L.), e as frutíferas abacateiro (Persea americana Mill.), caquizeiro (Diospyros kaki L.f.), morangueiro (Fragaria ananassa Duch.) e pessegueiro Prunus persica (L.) Batsch (Silva et al., 1968). 
H. indecisa é uma mariposa cujos adultos têm cerca de $5 \mathrm{~cm}$ de envergadura e $2 \mathrm{~cm}$ de comprimento (cabeça ao abdômen) (Rizzo, 1984). Nas fêmeas, as asas anteriores são de coloração cinza, com áreas delimitadas mais escuras, e as posteriores de coloração escura, com os bordos branco-amarelados, enquanto nos machos as asas posteriores são brancas. As cores das asas constituem o fenômeno chamado de aposematismo, uma forma de advertência para os seus inimigos (Grant, 2007). Além da cor das asas, as fêmeas são maiores que os machos. O abdômen é de coloração cinza, com cinco manchas de cor laranja no dorso e seis nas laterais. As lagartas, no último estádio de desenvolvimento, chegam a medir cerca de $7 \mathrm{~cm}$ de comprimento e possuem o corpo recoberto por espinhos de coloração marromavermelhados.

O estabelecimento de estratégias de controle de pragas envolve, entre outros conhecimentos, a bioecologia das espécies e a possibilidade de criá-las, de preferência, em dietas artificiais. Nos últimos 50 anos, o desenvolvimento de técnicas de criação de insetos, em dieta artificial, permitiu grande avanço no manejo de pragas, com a implementação de programas de criação massal para estudos de controle. Entre os insetos com maior sucesso de criação em dieta artificial, destacam-se os da ordem Lepidoptera com $40 \%$ do total dos grupos taxonômicos criados (Singh, 1977; Parra, 2000). As famílias Noctuidae e Tortricidae estão entre as que apresentam maior número de dietas para criação. Outras famílias, entretanto, como Arctiidae, possuem poucas espécies criadas em dieta artificial (Singh, 1977), o que dificulta o estabelecimento de novas medidas de controle, tais como a utilização de feromônio sexual e o controle biológico.

Embora $H$. indecisa tenha sido relatada como praga do milho em 1968 (Silva et al., 1968), pouco se conhece sobre sua biologia, ecologia e controle.

O objetivo deste trabalho foi estudar a biologia e elaborar a tabela de vida de fertilidade de $H$. indecisa, em dieta artificial, para sua criação em laboratório.

\section{Material e Métodos}

Os estudos foram realizados no Laboratório de Entomologia, Embrapa Clima Temperado, sob condições controladas de temperatura $\left(25 \pm 2^{\circ} \mathrm{C}\right)$, umidade relativa $(70 \pm 20 \%)$ e fotófase (14 horas).
As lagartas de $H$. indecisa foram coletadas em lavouras de milho, cultivadas em solos de várzeas em Pelotas, RS, a $31^{\circ} 46^{\prime} 19^{\prime \prime} \mathrm{S}, 52^{\circ} 20^{\prime} 33^{\prime \prime} \mathrm{W}$, e mantidas em laboratório em dieta artificial por duas gerações, quando foi, então, realizado o estudo da biologia.

No laboratório, as lagartas foram colocadas em caixas de gerbox ( $30 \mathrm{~cm}$ de comprimento $x 10 \mathrm{~cm}$ de largura x $6 \mathrm{~cm}$ de altura) e alimentadas com folhas de milho, até a fase pupal. Posteriormente, as pupas foram individualizadas, separadas por sexo, com base na abertura genital, e colocadas no interior de placas de acrílico, com algodão embebido em água, para manter a umidade. Após a emergência, casais foram colocados em gaiolas de PVC $(10 \mathrm{~cm}$ de altura $\times 10 \mathrm{~cm}$ de diâmetro). A parte interna das gaiolas foi revestida com papel jornal, e os adultos foram alimentados com solução de mel a $10 \%$, fornecido por capilaridade em pequenos recipientes de vidro, por meio de rolo dental. $O$ papel que revestia a gaiola internamente e que serviu de local para a postura, foi substituído diariamente, e o alimento foi trocado a cada dois dias.

$\mathrm{Na}$ criação em dieta artificial, foram utilizados tubos de vidro (2,3 cm de diâmetro $\times 9 \mathrm{~cm}$ de altura), tampados com algodão hidrófobo, previamente esterilizados a $180^{\circ} \mathrm{C}$. A dieta utilizada foi a de Burtton \& Perkins (1972), composta de: $247,5 \mathrm{~g}$ de feijão; $118,8 \mathrm{~g}$ de germe de trigo; 75,75 $\mathrm{g}$ de levedura de cerveja; 7,65 g de ácido ascórbico; 2,47 g de ácido sórbico; 4,72 g de metil parahidroxibenzoato (nipagin); $4,70 \mathrm{~mL}$ de formaldeído (38\%); 30,75 g de ágar e 1.792,5 mL de água. O preparo da dieta artificial e a técnica de criação foram realizados de acordo com Parra (2000).

Para o estudo da biologia, foram utilizadas 150 lagartas criadas individualmente em tubos de vidro, com $30 \mathrm{~mL}$ de dieta artificial. Foram avaliadas as seguintes variáveis: duração e viabilidade das fases de lagarta e de pupa, e o peso de pupas com 24 horas (machos e fêmeas). Para avaliação da fase adulta, foram formados 20 casais de ate 48 horas, para determinação da longevidade de machos e fêmeas, fecundidade e duração do período de pré-oviposição e oviposição. Também foi determinada a duração e a viabilidade do período embrionário, com 150 ovos coletados da segunda postura. Para a fase de pupa, foram 138 repetições.

A partir dos dados de duração do período de desenvolvimento (ovo-adulto), viabilidade total, razão sexual, período de pré-oviposição, número de ovos 
por dia e mortalidade diária de machos e fêmeas, foi elaborada uma tabela de vida de fertilidade, para quantificar a capacidade de crescimento de $H$. indecisa em cada geração, segundo Silveira Neto et al. (1976). Foram determinados, também, o número e a duração dos estádios, tendo-se realizado a medição diária da largura da cápsula cefálica de 20 lagartas, com uma ocular micrométrica acoplada a um microscópio estereoscópico. Para a determinação do número de estádios, foi utilizada a curva multimodal de freqüência de medidas de cápsula cefálica, com hipóteses testadas no modelo linearizado da regra de Dyar, por meio do programa Mobae (Modelos Bioestatísticos para a Entomologia) (Haddad et al., 1995).

Os dados do peso de pupas e de longevidade de machos e de fêmeas foram submetidos à análise de variância, e as médias foram comparadas pelo teste de Tukey, a 5\% de probabilidade.

\section{Resultados e Discussão}

A duração média da fase de ovo foi de $6 \pm 0,6$ dias (variação de 5-7 dias), e a viabilidade foi de $92,7 \pm 5,2 \%$ (variação de $80-100 \%$ ). As fases larval e pupal apresentaram duração de $25,4 \pm 3,1$ dias (variação de 21-54 dias) e 64,3 $\pm 25,3$ dias (variação de 14-133 dias), respectivamente, e a viabilidade foi de $92 \pm 6,76 \%$ (variação $80-100 \%$ ) e $71,9 \pm 21,1 \%$ (variação 42,85-100\%), respectivamente. O ciclo biológico (ovo-adulto) foi de 95,7 dias, e a viabilidade total de $61,3 \%$.

De maneira geral, os dados de duração das fases do ciclo biológico foram próximos aos registrados para outras espécies de Arctiidae, e as variações decorreram, provavelmente, da dieta utilizada para o desenvolvimento larval (Gomi et al., 2005, 2006). Apesar de a viabilidade do ciclo biológico (ovo-adulto) ter sido inferior aos $75 \%$, preconizados por Singh (1983) para que a dieta artificial seja considerada adequada, pode ocorrer uma estabilização e o aumento deste valor, a partir da sétima geração, em razão da adaptação dos insetos à referida dieta (Parra, 2000). Embora os valores de duração sejam característicos de cada espécie de Arctiidae, destaca-se a longa duração da fase pupal (64,3 dias), assim como sua grande variação (14 a 133 dias).

Durante o desenvolvimento larval, foram observados seis estádios (Figura 1); a duração de cada um foi, respectivamente: $4,2 \pm 0,1,3,3 \pm 0,1,4,0 \pm 0,1,4,1 \pm 0,2$, $3,5 \pm 0,2$ e 6,3 $\pm 0,2$ dias. A razão entre um estádio e o anterior correspondeu à regra de Dyar (Dyar, 1890), ou seja, a largura das cápsulas cefálicas aumentou em média, a cada estádio, à razão de 1,81 (a regra prevê variação de 1,1 a 1,9 , com média de 1,4$)$. O número de estádios larvais para Arctiidae varia de cinco a sete, em diferentes espécies (Betzholtz, 2003; Gomi et al., 2003; Rodríguez Loeches \& Barro, 2008), e está, portanto, dentro desse intervalo, já que no presente trabalho, H. indecisa apresentou seis estádios.

O peso de pupas foi de $0,73 \pm 0,08 \mathrm{~g}$ em machos e de $1,0 \pm 0,13 \mathrm{~g}$ em fêmeas, e diferiu significativamente entre sexos. Normalmente, em Lepidoptera, as fêmeas são mais pesadas do que os machos, pelo fato de adquirirem mais nutrientes, visto que são responsáveis pela reprodução (Slansky Junior \& Scriber, 1985).

O período de pré-oviposição foi de $2 \pm 0,2$ dias, e o de oviposição de $8,3 \pm 0,5$ dias e, nesse período, as fêmeas colocaram em média $1.531,5 \pm 243$ ovos. A longevidade de machos e fêmeas foi de $21,9 \pm 7,9$ e $21,8 \pm 6,1$ dias, respectivamente, e a razão sexual foi $0,5 \pm 0,1$. Os dados de longevidade e fecundidade de $H$. indecisa foram próximos aos de outras espécies de Arctiidae, como Halysidota caryae Harris (Lawrence, 1992), Amsacta moorei Butler (Saini, 1993) e Utetheisa ornatrix (L.) (Signoretti et al., 2008). Tais diferenças podem ser atribuídas à dieta utilizada no desenvolvimento da fase larval e à própria espécie, uma vez que essas variáveis biológicas são características de cada organismo.

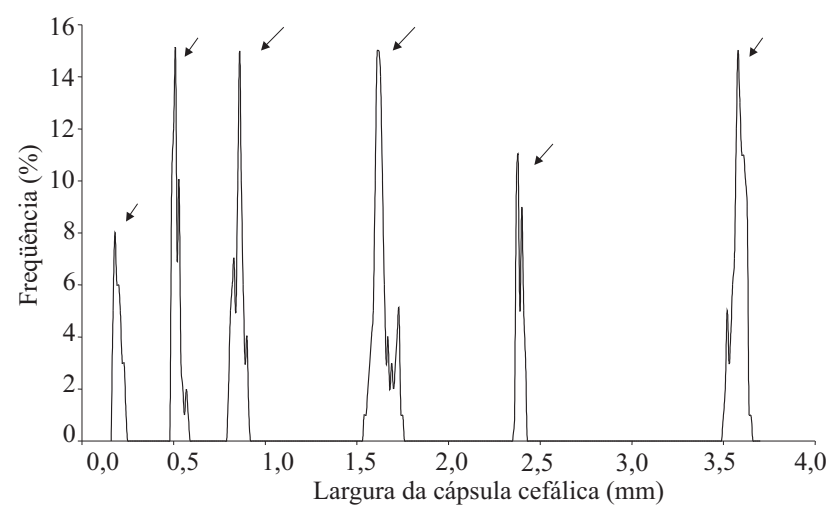

Figura 1. Distribuição de freqüência das medidas de cápsulas cefálicas de Hypercompe indecisa, criada em dieta artificial. Temperatura, $25 \pm 2^{\circ} \mathrm{C}$; umidade relativa do ar, $70 \pm 20 \%$; fotófase, 14 horas. As setas indicam os estádios da espécie. 
Determinou-se, pela taxa líquida de reprodução $\left(\mathrm{R}_{\mathrm{o}}\right)$, que $H$. indecisa tem capacidade de aumento de 283 vezes a cada geração, em laboratório, o que a caracteriza como espécie com elevado potencial biótico. Foi constatada, também, a duração média de 98 dias da geração (T), isto é, o tempo que decorre do nascimento dos pais ao nascimento dos seus descendentes. A taxa intrínseca de crescimento $\left(\mathrm{r}_{\mathrm{m}}\right)$, característica da espécie, foi de 0,058 , e a razão finita de aumento $(\lambda)$, ou seja, o número de fêmeas adicionadas à população por fêmea que dará fềmea, foi de 1,06. Esses resultados indicam que, decorridos 98 dias (duração média da geração), podem-se esperar cerca de 283 fêmeas resultantes de cada fêmea em fase de reprodução, o que demonstra o grande potencial de reprodução de $H$. indecisa, em condições de laboratório. A taxa máxima de aumento populacional ocorreu no 970 dia, que corresponde ao ponto de encontro da fertilidade específica $(\mathrm{mx})$ e da taxa de sobrevivência (lx) (Figura 2).

Embora o trabalho tenha sido realizado em laboratório, em condições constantes de temperatura, umidade e fotoperíodo, em campo o inseto pode sofrer adversidades, em razão de fatores bióticos (parasitóides, predadores e patógenos) e abióticos (clima, alimento etc.), e sua descendência pode reduzir-se; assim, para a avaliação desses fatores ecológicos, seriam necessários estudos de tabela de vida ecológica, como foi feita para Diatraea saccharalis (Fabr.) em cana-de-açúcar (Botelho, 1985).

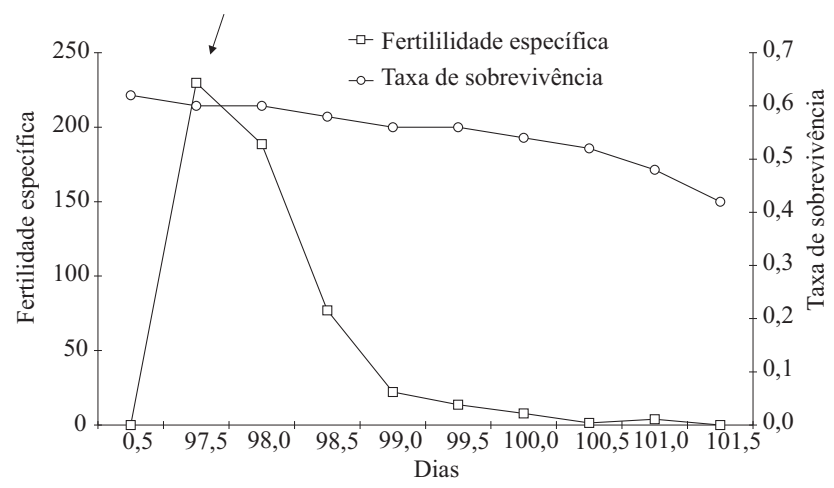

Figura 2. Relação entre fertilidade específica $(\mathrm{mx})$ e taxa de sobrevivência (lx) de Hypercompe indecisa, criada em dieta artificial. A seta indica a taxa máxima de aumento. Temperatura, $25 \pm 2{ }^{\circ} \mathrm{C}$; umidade relativa do ar, $70 \pm 20 \%$; fotófase, 14 horas.

\section{Conclusão}

Hypercompe indecisa apresenta grande potencial de reprodução, em condições de laboratório, e a dieta artificial é adequada para a manutenção de sua criação.

\section{Agradecimentos}

À estagiária Daiane de Almeida e à técnica de laboratório Ladi Macedo Viana, pelo auxílio na realização do trabalho.

\section{Referências}

BETZHOLTZ, P.E. The discrepancy between food plant preference and suitability in the moth Dysauxes ancilla. Web Ecology, v.4, p.7-13, 2003.

BOTELHO, P.S.M. Tabela de vida ecológica e simulação da fase larval da Diatraea saccharalis (Fabr., 1794) (Lep.: Pyralydae). 1985. 110p. Tese (Doutorado) - Escola Superior de Agricultura Luiz de Queiroz, Piracicaba.

BURTTON, R.L.; PERKINS, W.D. WSB: a new laboratory diet for the corn earworm and the fall armyworm. Journal of Economic Entomology, v.65, p.385-386, 1972.

DYAR, H.G. The number of molts of lepidopterous larvae. Psyche, v.5, p.420-433, 1890.

GALLO, D.; NAKANO, O.; SILVEIRA NETO, S.; CARVALHO, R.P.L.; BAPTISTA, G.C.; BERTI FILHO, E.; PARRA, J.R.P.; ZUCCHI, R.A.; ALVES, S.B.; VENDRAMIM, J.D.; MARCHINI, L.C.; LOPES, J.R.S.; OMOTO, C. Entomologia Agrícola. Piracicaba: Fealq, 2002. 920p.

GOMI, T. Sexual difference in the effect of temperature on the larval development in Hyphantria cunea (Drury) (Lepidoptera: Arctiidae). Applied Entomological Zoology, v.41, p.303-307, 2006.

GOMI, T.; HIROCHIKA, M.; NAGASAKA, M.; HAGIHARA, H.; FUKUDA, T. Effects of diet on life-history traits in a trivoltine Kobe population of Hyphantria cunea (Drury) (Lepidoptera: Arctiidae). Applied Entomological Zoology, v.40, p.475-482, 2005.

GOMI, T.; MITSUNORI, I.; YAMADA, D. Local divergence in developmental traits within a trivoltine area of Hyphantria cunea Drury (Lepidoptera: Arctiidae). Entomological Science, v.6, p.71-75, 2003.

GRANT, J.B. Ontogenetic colour change and the evolution of aposematism: a case study in panic moth caterpillars. Journal of Animal Ecology, v.3, p.439-447, 2007.

HADDAD, M.L.; MORAES, R.C.B.; PARRA, J.R.P. Programa MOBAE: modelos bioestatísticos aplicados à entomologia (software). Piracicaba: Esalq-USP, 1995. 44p. 1 disquete.

LAWRENCE, W.S. The effects of group size and host species on development and survivorship of a gregarious caterpillar Halisidota caryae (Lepidoptera: Arctiidae). Ecological Entomology, v.15, p.53-62, 1990. 
PARRA, J.R.P. Técnicas de criação de insetos para programas de controle biológico. Piracicaba: Fealq, 2000. 137p.

RIZZO, H.F.R. Gata peluda de los almácigos, Ecpantheria indecisa Walker (Lepidoptera: Arctiidae). In: INTEGRACIÓN investigación y extensión rural. Buenos Aires: Inta, 1984. 57p.

RODRÍGUEZ LOECHES, L.; BARRO, A. Life cycle and immature of the arctiid moth, Phoenicoprocta capistrata. Journal of Insect Science, v.8, p.1-13, 2008.

SAINI, R.K. Development and survival of red hairy caterpillar, Amsacta moorei Butler on some cultivated plants and weeds in Haryana. Journal of Insect Science, v.6, p.64-68, 1993.

SANCHEZ SOTO, S.; NAKANO, O. Novo registro de Lepidoptera na cultura do milho no Brasil. Neotropical Entomology, v.32, p.365-366, 2003.

SIGNORETTI, A.G.C.; NAVA, D.E.; BENTO, J.M.S.; PARRA, J.R.P. Biology and thermal requirements of Utetheisa ornatrix (L.) (Lepidoptera: Arctiidae) reared on artificial diet. Brazilian Archives of Biology and Technology, v.51, p.447-453, 2008.
SILVA, A.G.A.; GONÇALVES, C.R.; GALVÃO, D.M.; GONÇALVES, A.J.L.; GOMES, J.; SILVA, M.N.; SIMONI, L. Quarto catálogo dos insetos que vivem nas plantas do Brasil. Seus parasitos e predadores, insetos hospedeiros e inimigos naturais. Rio de Janeiro: Ministério da Agricultura, 1968. 622p. Parte II, Tomo 1.

SILVEIRA NETO, S.; NAKANO, O.; BARBIN, D.; VILLA NOVA, N.A. Manual de ecologia dos insetos. São Paulo: Ceres, 1976. 419p.

SINGH, P. A general purpose laboratory diet mixture for rearing insects. Insect Science and Its Application, v.4, p.357-362, 1983. SINGH, P. Artificial diets for insects, mites, and spiders. New York, Plenum, 1977. 594p.

SLANSKY JUNIOR, F.; SCRIBER, J.M. Food consumption and utilization. In: KERKUT, G.A.; GILBERT, L.I. (Ed.). Comprehensive insect physiology, biochemistry, and pharmacology. Oxford: Pergamon Press, 1985. p.87-183.

Recebido em 8 de agosto de 2008 e aprovado em 11 de novembro de 2008 